Supporting Information for:

\title{
Nanoscale transient magnetization gratings created and probed by femtosecond extreme ultraviolet pulses
}

Dmitriy Ksenzov ${ }^{*}{ }^{1}$, Alexei A. Maznev ${ }^{2}$, Vivek Unikandanunni ${ }^{3}$, Filippo Bencivenga ${ }^{4}$, Flavio Capotondi ${ }^{4}$, Antonio Caretta ${ }^{4}$, Laura Foglia $^{4}$, Marco Malvestuto $^{4}$, Claudio Masciovecchio $^{4}$, Riccardo Mincigrucci ${ }^{4}$, Keith A. Nelson ${ }^{2}$, Matteo Pancaldi ${ }^{3}$, Emanuele Pedersoli $^{4}$, Lisa Randolph $^{1}$, Hendrik Rahmann ${ }^{1}$, Sergei Urazhdin ${ }^{5}$, Stefano Bonetti ${ }^{3,6}$, and Christian Gutt ${ }^{1}$

\section{Affiliations}

${ }^{1}$ Department Physik, Universität Siegen, Walter-Flex-Str. 3, 57072, Siegen, Germany

${ }^{2}$ Department of Chemistry, Massachusetts Institute of Technology, 77 Massachusetts Avenue, Cambridge, MA 02139, United States

${ }^{3}$ Department of Physics, Stockholm University, 10691 Stockholm, Sweden

${ }^{4}$ Elettra Sincrotrone Trieste S.C.p.A., Strada Statale 14, km 163.5, 34149 Basovizza (TS), Italy.

${ }^{5}$ Department of Physics, Emory University, Atlanta, Georgia 30322, United States

${ }^{6}$ Department of Molecular Sciences and Nanosystems, Ca' Foscari University of Venice, 30172 Venice, Italy

* Email: dmitriy.ksenzov@uni-siegen.de (D.K.) 


\section{S1. Sample preparation}

The metal stack was deposited on a 100 nm-thick $\mathrm{Si}_{3} \mathrm{~N}_{4}$ membrane by sputtering at room temperature in 4.9 mTorr of ultrapure Ar, in an ultrahigh vacuum chamber with a base pressure of $6 \times 10^{-9}$ Torr. The $9 \mathrm{~nm}$-thick $\mathrm{Co}_{81} \mathrm{Gd}_{19}$ film was deposited by co-sputtering from pure $\mathrm{Co}$ and $\mathrm{Gd}$ targets, with respective deposition rates calibrated by a quartz crystal microbalance to the precision of better than $0.1 \% .4 \mathrm{~nm}$-thick Ta/5nm-thick Pt bilayer was used as a buffer layer. The film was protected from oxidation by a $4 \mathrm{~nm}$-thick Ta capping layer. The deposition rates for $\mathrm{Ta}, \mathrm{Pt}$, and CoGd were $0.2 \mathrm{~A} / \mathrm{s}, 1 \mathrm{~A} / \mathrm{s}$, and $0.4 \mathrm{~A} / \mathrm{s}$, respectively.

\section{S2. Magnetic properties}

The magnetic properties of the sample were characterized by vibrating sample magnetometry at room temperature. Figure S1 shows In-plane (IP) and Out-of-plane (OOP) magnetization hysteresis loops. From the hysteresis loops, we obtain the value of the saturation magnetization $\mathrm{M}_{\mathrm{s}}$ (magnetic moment/volume) to be $250 \mathrm{emu} / \mathrm{cm}^{3}$. The OOP saturation field is around $0.15 \mathrm{kOe}$, whereas the IP saturation field is around $0.3 \mathrm{kOe}$. We estimate the anisotropy field $H_{k}$, related to the IP saturation field $\mathrm{H}_{\mathrm{s}}$, by the following expression, $H_{k}=H_{s}+4 \pi M_{s}$. This gives the value of $H_{k}$ as $3.44 \mathrm{kOe}$ and subsequently we estimate the anisotropy constant $K_{u}=H_{k} M_{s} / 2=4.3 \times 10^{5}$ $\mathrm{erg} / \mathrm{cm}^{3}$. The estimated values are close to the ones found in literature for similar samples (refs. S1, S2).

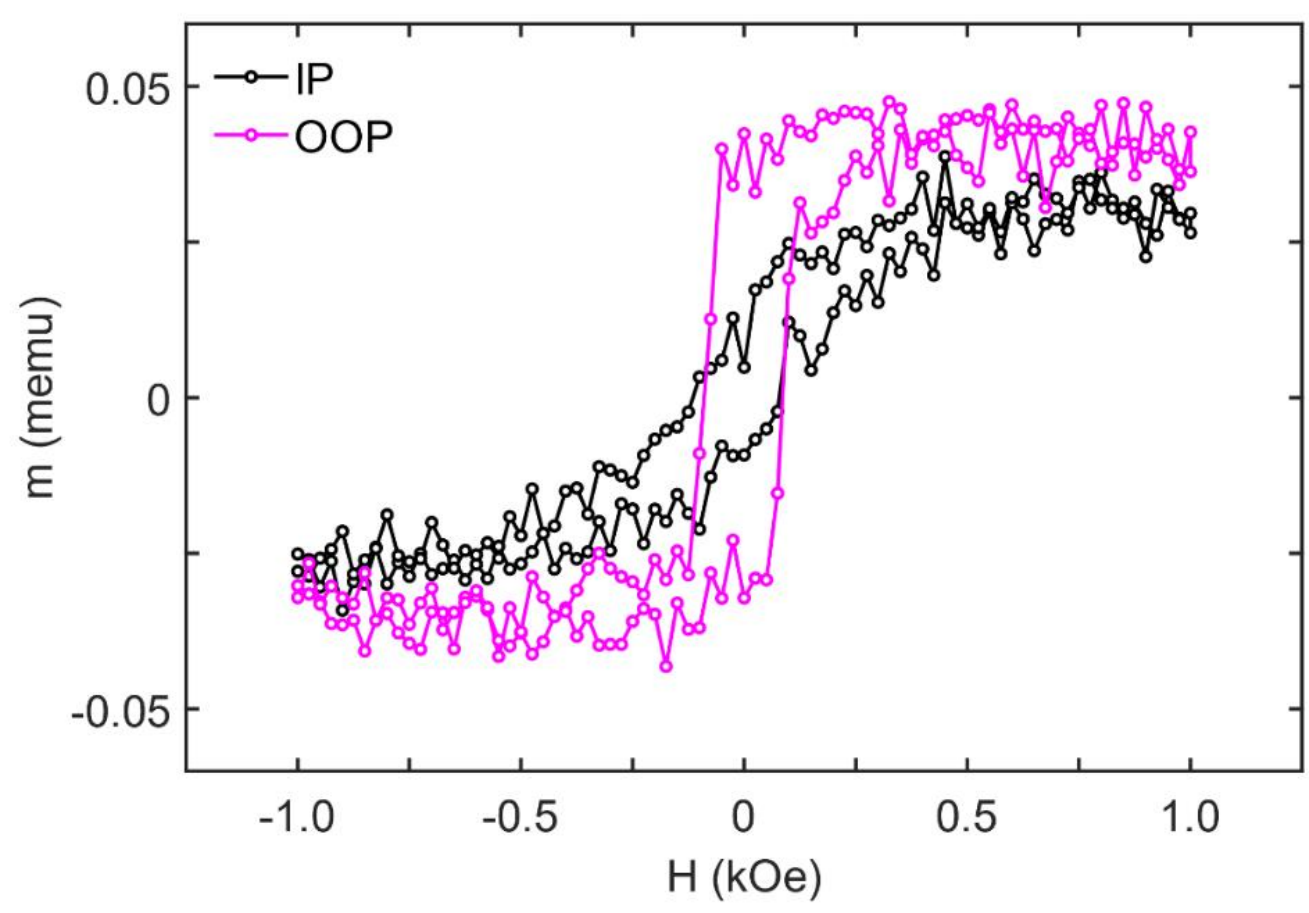

Figure S1. In-plane (IP) and out-plane (OOP) magnetization hysteresis loops obtained for the CoGd sample. 


\section{S3. Experimental details}

The experiments were performed at the EIS-TIMER end-station (ref. 26). This setup uses wavefront division beam-splitting to produce three FEL beams which are overlapped at the sample. The parameters of pump and probe pulses are listed in Table S1. Further details of the optical setup can be found in ref. 26. A holey electromagnet is used to supply a permanent magnetic field orthogonal to the sample surface while still allowing all the EUV beams to reach the sample.

Table S1. Parameters of the pump and probe beams for both configurations.

\begin{tabular}{l|c|c}
\hline & Configuration 1 & Configuration 2 \\
\hline Grating period $\Lambda, \mathrm{nm}$ & 43.6 & 87.2 \\
\hline Pump beam: & 20.8 & 41.6 \\
Wavelength, $\mathrm{nm}$ & $2 \times 1.1$ & $2 \times 2.0$ \\
Pulse energy, $\mu \mathrm{J}$ & & \\
\hline Probe beam: & 20.8 & 20.8 \\
Wavelength, $\mathrm{nm}$ & 0.17 & 0.14 \\
Pulse energy, $\mu \mathrm{J}$ &
\end{tabular}

The diffracted probe pulse is detected by a soft x-ray in-vacuum charge-coupled device (CCD) camera (Princeton PI-MTE). The EUV TG signal was determined by integrating the CCD counts in a region of interest around the signal beam. Normalization to the cube of the FEL pulse energy was used to reduce the noise caused by the FEL fluctuations. To convert the counts recorded by the CCD into incoming photon counts we used a conversion coefficient of 5.8 photons per CCD count, which includes the detector quantum efficiency.

\section{S4. Diffraction of the probe beam by a magnetization grating}

For the probe beam directed along the sample normal $z$ (we neglect the small incidence angle) and polarized along $y$, the electric field is given by $E_{0} \boldsymbol{e}_{y} \exp (i k z-i \omega t)$, where $\boldsymbol{e}_{y}$ is the unit Jones vector, and $k=2 \pi / \lambda$ is the wavevector magnitude. The linearly polarized light can be decomposed into the two circularly polarized components:

$$
\boldsymbol{e}_{y}=\frac{i}{\sqrt{2}}\left(\boldsymbol{e}_{-}+\boldsymbol{e}_{+}\right), \text {where } \boldsymbol{e}_{ \pm}=\frac{1}{\sqrt{2}}\left(\begin{array}{c}
1 \\
\pm i
\end{array}\right)
$$

We consider a weak grating of dichroism given by Eq. (2) from the main text,

$$
\delta n_{ \pm}= \pm(\Delta \delta+i \Delta \beta) \frac{\Delta}{2} \cos q x
$$

As long the diffraction efficiency is low, the diffracted field amplitude for each of the circularly polarized components is given by

$$
\boldsymbol{E}_{d \pm}=E_{0} \frac{i}{\sqrt{2}} \boldsymbol{e}_{ \pm} \frac{k d \Delta}{4}( \pm i \Delta \delta \mp \Delta \beta) \exp [-k d(\beta \pm \Delta \beta)],
$$

where $d$ is the thickness of the grating. 
Assuming that $k d \Delta \beta<<1$, the amplitude of the diffracted electric field is

$$
\boldsymbol{E}_{d}=\left(\boldsymbol{E}_{d-}+\boldsymbol{E}_{d+}\right)=E_{0} \boldsymbol{e}_{x} \frac{k d \Delta}{4}(-i \Delta \delta+\Delta \beta) \exp [-k d \beta],
$$

with the polarization orthogonal with respect to that of the incident probe beam. The diffraction efficiency, i.e., the ratio of the diffracted intensity to the intensity of the transmitted zeroth-order beam, is given by

$$
\frac{I_{d}}{I_{0}}=\frac{k^{2} d^{2}}{16} \Delta^{2}\left(\Delta \delta^{2}+\Delta \beta^{2}\right) .
$$

\section{S5. Absorption profile of the multilayered sample}

Computations of the absorption profile of the multilayered sample are based on the procedure described in the ref. S3. and use of the atomic scattering factors (ref. S4) for the refractive indices of the layers (Table S2).

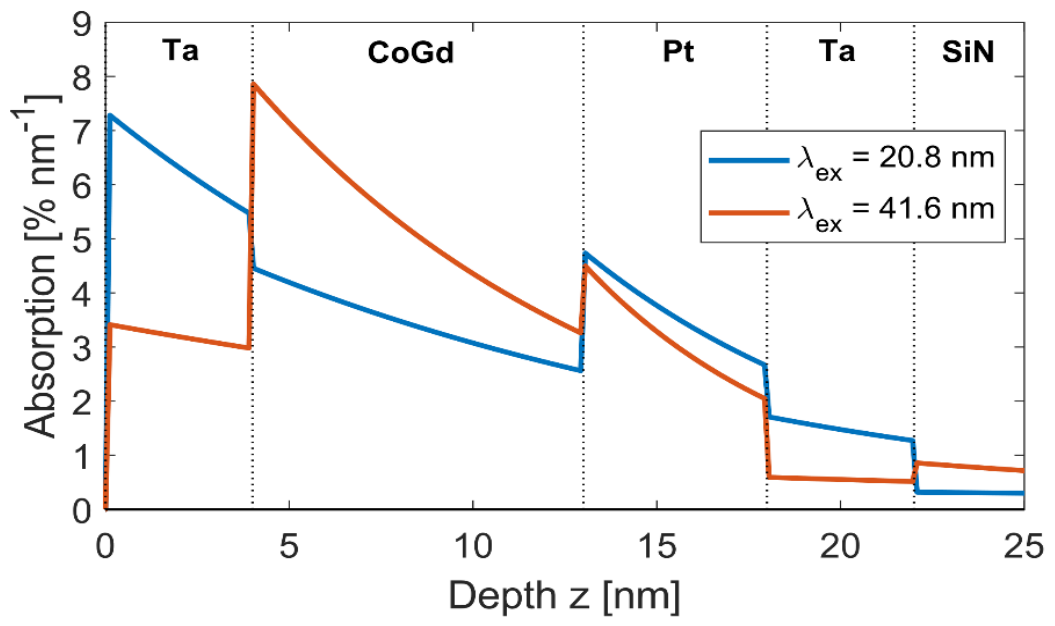

Figure S2. Calculated absorption profiles as a function of depth inside the multilayer for the pump beam with wavelengths $\lambda_{\mathrm{ex}}=20.8$ and $41.6 \mathrm{~nm}$.

Table S2. Parameters of multilayer used for the calculation of the absorption profiles.

\begin{tabular}{cccccc}
\hline \multirow{2}{*}{ Layer } & \multirow{2}{*}{$\mathrm{d}, \mathrm{nm}$} & \multicolumn{2}{c}{$20.8 \mathrm{~nm}$} & \multicolumn{2}{c}{$41.6 \mathrm{~nm}$} \\
\cline { 3 - 6 } & & $n$ & $k$ & $n$ & $k$ \\
\hline $\mathrm{Ta}$ & 4 & 0.853 & 0.120 & 0.898 & 0.114 \\
$\mathrm{CoGd}$ & 9 & 0.973 & 0.099 & 0.832 & 0.315 \\
$\mathrm{Pt}$ & 5 & 0.918 & 0.188 & 0.746 & 0.515 \\
$\mathrm{Ta}$ & 4 & 0.853 & 0.120 & 0.898 & 0.114 \\
$\mathrm{SiN}$ & 100 & 0.925 & 0.030 & 0.747 & 0.194 \\
\hline
\end{tabular}

\section{S6. Magneto-optical constants for $\mathrm{Co}_{0.81} \mathbf{G d}_{0.19}$ alloy}

Assuming that the magneto-optical (MO) constants $\Delta \delta$ and $\Delta \beta$ at the Co M edge are determined by the number of Co atoms per unit volume, the MO values for Co (ref. 30) should be multiplied by 0.59 to get the $\mathrm{MO}$ constants of CoGd: 


$$
\Delta \delta_{C O G d}\left(\Delta \beta_{C o G d}\right)=0.81 \frac{n_{a}^{C o G d}}{n_{a}^{C o}} \Delta \delta_{C o}\left(\Delta \beta_{C o}\right)=0.59 \Delta \delta_{C o}\left(\Delta \beta_{C o}\right),
$$

where $n_{a}=\rho N_{a} / M$ is the number density, $\rho$ is the physical density, $N_{a}$ is the Avogadro constant, and $M$ is molar mass. At the $20.8 \mathrm{~nm}$ (or $59.6 \mathrm{eV}$ ), the MO constants for Co0.81 $\mathrm{Gd} 0.19$ alloy are $\Delta \delta \approx$ $8.8 \cdot 10^{-3}$ and $\Delta \beta \approx-4.7 \cdot 10^{-3}$.

\section{S7. Temperature grating amplitude}

Below the Curie temperature, the magnetization is usually described in terms of Bloch's law, $M(T)=M_{S}(0) \times\left(1-\left(T / T_{C}\right)^{\alpha}\right)$, where $M_{s}(0)$ is the saturation magnetization at $0 \mathrm{~K}$ and $T_{C}$ is the Curie temperature. The exponent $\alpha$ is known as the Bloch's exponent, $\alpha=3 / 2$ for bulk materials. The Curie temperature for the $\mathrm{Co} 0.81 \mathrm{Gd} 0.19$ alloy is about $900 \mathrm{~K}$ (ref. 33). Based on the estimated 13$21 \%$ peak-to-peak magnetization grating amplitude as a fraction of the saturation magnetization at $300 \mathrm{~K}$, we estimated that the peak-to-peak temperature grating amplitude was $110 \mathrm{~K}(180 \mathrm{~K})$ for $\Lambda=43.6 \mathrm{~nm}(87.2 \mathrm{~nm})$.

\section{S8. Visibility of interference fringes and its effect on the diffraction efficiency}

The spectrum of $20.8 \mathrm{~nm}$ pump pulses was monitored during the experiment. The shape of the distribution was close to Gaussian with a full width at half maximum (FWHM) $\Delta v=8.9 \mathrm{THz}$, which is only $20 \%$ larger than the transform-limited bandwidth. The visibility of the interference fringes (i.e. the difference between minimum and maximum fluence given the same average fluence) resulting from two pulses with a Gaussian spectrum separated by a time interval $\tau$ is given by (ref. S5)

$$
V=e^{-\pi^{2} \tau^{2} \Delta v^{2} / 2 \sqrt{\ln 2}}
$$

The time delay between pump pulses crossed at the angle $2 \Theta$ is given by

$$
\tau=\frac{2 x}{c} \tan \Theta,
$$

where $c$ is the speed of sound and $x$ is the distance from the point where the pulses overlap perfectly (ideally, at the center of the pump spot) along the $x$ axis in Fig. 1A. Thus the visibility is given by $V=e^{-x^{2} / b^{2}}$, where

$$
b=\frac{\sqrt{\ln 2} c}{\pi \Delta v \tan \Theta} .
$$

With the measured value of $\Delta v$ we get $b=36 \mu \mathrm{m}$ for $20.8 \mathrm{~nm}$ excitation. For $41.6 \mathrm{~nm}$ excitation the spectrum of the pump pulses was not monitored, but we believe that its FWHM was similar since FEL pulses are close to transform-limited (ref. 29).

Let us now consider the effect of the width of the pump interference pattern and the EUV spot size on the measured diffraction efficiency. We assume that the excitation and probe spots are Gaussian (admittedly, a very crude approximation), with $1 / e$ spot size radii $a_{e x}$ and $a_{p}$, respectively. Then the amplitude of the excitation fluence grating is given by

$$
F_{T G}=F_{T G 0} e^{x^{2} / b^{2}-x^{2} / a_{e x}^{2}-y^{2} / a_{e x}^{2}}
$$


(note that the interference visibility only varies along $x$ ), and the probe fluence is given by

$$
F_{p}=F_{p 0} e^{-x^{2} / a_{p}^{2}-y^{2} / a_{p}^{2}}
$$

Since according to Eq. (S5) the diffraction efficiency $S=I_{d} / I_{0}$ is quadratic with respect to the magnetization grating amplitude, we assume it to be given by $\gamma F_{T G}^{2}$, where $\gamma$ is a constant. Then at the center of the spot

$$
S_{0}=\gamma F_{T G 0}^{2}
$$

whereas the total diffraction efficiency is given by

$$
S_{t o t}=\frac{\gamma \int F_{p} F_{T G}^{2} d x d y}{\int F_{p} d x d y}=\gamma F_{T G 0}^{2}\left(1+\frac{2 a_{p}^{2}}{a_{e x}^{2}}+\frac{2 a_{p}^{2}}{b^{2}}\right)^{-1 / 2}\left(1+\frac{2 a_{p}^{2}}{a_{e x}^{2}}\right)^{-1 / 2}
$$

The ratio is given by

$$
\frac{s_{0}}{s_{t o t}}=\left(1+\frac{2 a_{p}^{2}}{a_{e x}^{2}}+\frac{2 a_{p}^{2}}{b^{2}}\right)^{1 / 2}\left(1+\frac{2 a_{p}^{2}}{a_{e x}^{2}}\right)^{1 / 2}
$$

If $a_{e x}=a_{p}=a$, this simplifies to

$$
\frac{s_{0}}{s_{t o t}}=3^{1 / 2}\left(3+\frac{2 a^{2}}{b^{2}}\right)^{1 / 2}
$$

For $2 a=300 \mu \mathrm{m}$ and $b=36 \mu \mathrm{m}$, we get $S_{0} / S_{\text {tot }}=10.6$, i.e. the diffraction efficiency at the center of the spot is an order of magnitude higher than the measured total diffraction efficiency.

\section{Additional References}

S1. Cronemeyer, D. C. Perpendicular Anisotropy in Gd 1- x Co x Amorphous Films Prepared by RF Sputtering. AIP Conf Proc. 1974 18, 85. DOI: 10.1063/1.3141747

S2. Taylor, R. C., Gangulee. A. Magnetization and magnetic anisotropy in evaporated GdCo amorphous films. J. Appl. Phys. 1976 47, 4666. DOI: 10.1063/1.322397

S3. Khorsand, A., Savoini, M., Kirilyuk, A., Rasing. Th. Optical excitation of thin magnetic layers in multilayer structures. Nature Mater 2014 13, 101-102. DOI: 10.1038/nmat3850

S4. Henke, B.L.; Gullikson, E.M.; Davis, J.C. X-ray interactions: photoabsorption, scattering, transmission, and reflection at E=50-30000 eV, Z=1-92, At. Data Nucl. Data Tables 1993 54, 181-342, DOI: 10.1006/adnd.1993.1013

S5. Abdulhalim, I. Spatial and temporal coherence effects in interference microscopy and full-field optical coherence tomography, Ann. Phys. (Berlin) 2012 524, 787-804, DOI:

10.1002/andp. 201200106 